\title{
EI TALLER COMO ANTECEDENTE FORMATIVO DE LA ESCUELA DE TRABAJO SOCIAL DE LA UNIVERSIDAD DE COSTA RICA
}

\author{
Freddy Esquivel Corella ${ }^{I}$
}

\section{Resumen}

Este artículo expone críticamente las influencias latinoamericanas y nacionales que se entrecruzaron en el planteamiento del Taller como base pedagógica de la educación del Trabajo Social en la Universidad de Costa Rica en la década de los años setentas del siglo XX.EI Taller fue considerado como una estrategia de aprendizaje que integraría la teoría con la práctica, que estimularía el trabajo grupal de forma horizontal desafiando la clásica educación bancaria y estrecharía los vínculos de los y las estudiantes con los grupos populares.

Descriptores: Taller, Trabajo Social, Universidad de Costa Rica, Reconceptualización.

\section{Abstract}

This article discusses the national and Latin American influences in the approach of the Taller (Workshop)as a pedagogical foundation of Social Work education at the University of Costa Rica in the early seventies of the twentieth century.The Tallerwas seenasa learning strategythat would integratetheorywithpractice, participatorygroup work, andwould narrowlinks of the studentswiththe popular groups.

Keywords:Taller, Social Work, University of Costa Rica, Reconceptualization.

\section{Acotaciones previas}

La profesión de Trabajo Social en Costa Rica tiene sus umbrales educativos en 1942 cuando se funda la Escuela de Servicio Social, misma que pasa a institucionalizarse en 1944 en la recién inaugurada Universidad de Costa Rica (UCR) (Molina y Sáenz, 1994). Su integración a la UCR, como parte del proceso de reacomodo de los estudios superiores en el país, condujo a que se instalara como un Departamento de la Facultad de Ciencias Económicas en 1947 (Casas, 1975). En 1957 posterior a la fundación de la Segunda República liderada por la socialdemocracia, el cuerpo docente solicita al Consejo Universitario que se le otorgue el rango de Escuela Anexa, para salvaguardar independencia en la mayoría de sus decisiones formativas (Fallas, 2010). Para 1974 es ubicada en la inicial Facultad de Ciencias Sociales donde se integra a un conjunto de Escuelas de diferente procedencia, naturaleza y significado socio-histórico (Esquivel, 2007).

La formación del Trabajo Social costarricense tiene entre sus características: la constante y dinámica preocupación por perfeccionar la tarea educativa (Esquivel, 2002), la cual se perfiló 
por la búsqueda de incorporar las demandas de los entes contratantes de la época (Beeche, 1951); la solicitud de asesoría internacional (Mora, 1968); la madurez para crear espacios de autodefinición de orientación universitaria así como profesional (Guzmán, 1973); y la producción de conocimientos para fundamentar nuevos aportes a las cuestiones relacionadas con su desarrollo en la educación superior (Barboza, 1977). En esas configuraciones se propone la modalidad de los Tallerescomo estrategia pedagógicavital para la formación profesional enlas tres últimas décadas del siglo XX, asociada a las respuestas que se encontraban en el colectivo profesional ante las convulsiones latinoamericanas a las que el Trabajo Social se enfrentaba.

\section{El Taller fue definido como:}

[...] una instancia teórico-práctica, donde un grupo integrado por maestros y alumnos problematizan un aspecto de la realidad social, buscando su conexión estructural con una problemática más amplia, con el objeto de definir formas de transformación de dicha problemática y de su conexión estructural [...] (Quezada, 1995, p.12)

Por su lado, Romero (1988) hace referencia a que esta propuesta era considerada prácticamente como un método para la enseñanza de la profesión, textualmente señala:

[...] es un método que busca la praxis extendiendo ésta como la integración de la teoría y la práctica para transformar, conocer y actuar. Mediante el Taller se pretende evolucionar en el ámbito de la educación, para contribuir al desarrollo integral del ser humano. En el caso de la formación de trabajadores sociales, se pretende no sólo este desarrollo integral en los estudiantes y futuros profesionales, sino también en los grupos sociales, hacia los cuales se dirige la acción profesional. (p.4)

Sumado a ello, Campos, Molina, Molina, Romero y Ruiz (1977) lo explicaban como:

[...] una forma de aprender y enseñar haciendo, cuyo rasgo pedagógico másimportante se resume en el desarrollo de la capacidad de reflexión, sobre determinados aspectos de realidad social pero que al mismo tiempo, tal reflexión depende de las condiciones específicas en las que se desenvuelve el taller y de los propósitos y características que éste adopta [...] (p.205)

Estas autoras advierten que el Taller tuvo diferentes significados en América Latina, por ejemplo en Chile, Ecuador y El Salvador, consistía en un curso laboratorio, separado de los teóricos (Campos, Molina, Molina, Romero y Ruiz, 1977). No obstante, en la Escuela costarricense se buscó una mayor integración con la totalidad de los cursos. De acuerdo a Alfaro, Arroyo, Araya, Jackson, Rosales y Villalta (1981), el mismo surgió como una nueva modalidad que pretendía responder a los objetivos profesionales de participación, organización, movilización y concientización de los sectores subordinados, por su posición y situación de clase en cuanto a los medios de producción.

El Taller buscaba una integración de la investigación, la docencia y la acción social; en producción de conocimientos se suponía que impulsaba la captación progresiva y sistemática de las expresiones de la realidad particular, en la cual se desenvuelve la praxis (Alfaro, et al., 1981). En docencia era considerada como un momento de inversión de los insumos culturales, que favorecen un ordenamiento conceptual preliminar de la realidad; en extensión se materializaba la proyección de las actividades que realizan estudiantes y docentes; las modalidades de esta acción correspondían a los grados de formación que el Taller cubría, participando siempre en 
tareas socialmente útiles, generalmente relacionadas con las actividades asalariadas de las zonas geográficas, de manera que le permitiera al o la estudiante legitimarse ante el grupo y emprender progresivamente la superación de una acción espontánea a una acción organizativa (programada), además de fortalecer su formación profesional (Alfaro, et al., 1981). Las fuerzas del contexto histórico, las bases intelectuales que brindan respaldo al Taller, así como la creciente expansión de la UCR, se instituyen en una amalgama que consolidará este proyecto pedagógico como el más sostenido y defendido que ha conocido la formación académica en el Trabajo Social de este país.

\section{El impulso del Trabajo Social Latinoamericano}

Aproximadamente desde 1965 en el I Seminario Regional Latinoamericano de Servicio Social desarrollado en Porto Alegre, Brasil, se sientan las bases primigenias que impulsan lo que en Trabajo Social de América Latina se denominó Movimiento de Reconceptualización. Al respecto Mora, Castro, Araya y Vega (1983) anotan:

[...] la Reconceptualización se define como un proceso crítico de la trayectoria del trabajador social, de su papel como profesional, de las metodologías empleadas para realizar sus funciones y de los objetivos y objeto de la profesión, el Taller también se constituye en una metodología crítica respecto a la profesión y al proceso pedagógico formativo (p.10)

Por su lado, Guzmán (1973) afirma que de este proceso se empezó a gestar una conciencia de cambio en el gremio y la academia latinoamericana, impulsándose principalmente por las agendas de los Seminarios Latinoamericanos de Servicio Social. Guzmán (1973), también destaca entre otros esfuerzos los Documentos de Araxá en 1967 y Teresópolis en 1970, como intentos de socialización del debate provocado por este impulso crítico. En esos documentos se plantearon, según la autora, la necesidad de investigar, teorizar, enseñar y practicar un Trabajo Social latinoamericano, ello con el fin de estar en capacidad de dar una respuesta concreta a los problemas regionales.

La preocupación por la supuesta separación de la teoría y la práctica (herencia claramente funcionalista y con acento tendencial de la exigencia empírica), parecía ser el eje de las preocupaciones de quienes se encontraban luchando por un ejercicio profesional más articulado a las demandas de intervención y de las expresiones de la "cuestión social", entendida como un conjunto de expresiones de luchas sociales ante las condiciones de vida de la clase trabajadora que reflejaban la expoliación y el pauperismo social (Yazbek, 2000).Tal y como afirma Parra (2005), dicho movimiento sentó las bases para la constitución de nuevos proyectos profesionales en el Trabajo Social, abriendo un amplio abanico de posturas y tendencias en el desarrollo de esta profesión en América Latina.

Por otro lado, este movimiento se caracterizó por una ausencia de homogeneidad, diversidad de asociación a diversos planteamientos societarios, posturas modernizadoras, critica (a veces poco sustentada) a los planteamientos conservadores que impregnaban la profesión, incorporación poco fiel de tradiciones teóricas, tornándolas en sustentos mecanicistas propio de lecturas someras de los textos originales, o bien como pasó con el marxismo de manuales de interpretación de los clásicos (Retana,2002; Araya, Calderón, Chinchilla y Vega,1986). El tema de las prácticas académicas estuvo puesto en debate enese contexto. Sobre ese particular, el Centro Latinoamericano de Trabajo Social - CELATS (1988), afirmaba que: "A partir de los años '70, al calor de 
la Reconceptualización, muchas escuelas comenzaron a transitar por el camino de los llamados Talleres, como una forma de realizar las prácticas pre-profesionales de sus alumnos" (p.8).

Es posible afirmar que esa propuesta no tuvo su desarrollo y fundamento por igual en los diferentes países en que se incorpora, por ejemplo se citan datos de escuelas hondureñas, peruanas, nicaragüenses, salvadoreñas, guatemaltecas, argentinas, ecuatorianas y costarricenses; siendo sus protoformas ciertas unidades académicas de Chile - especialmente la Universidad Católica de Chile y de Valparaíso (ver Aylwin y Gissi, 1978). Por ejemplo, Araneda (2005) afirma que en Ecuador, bajo la influencia de la profesora Aylwin, de la Universidad Católica de Chile, se desarrolló esta propuesta. A su vez en Guatemala, Tomasa de León (2005) escribe que gracias al soporte chileno, es que surge y se desarrolla la concepción de Taller como instancia de reflexión teórica y de la realidad.

En el caso costarricense Guzmán (1978) da crédito de la influencia que recibió de otras latitudes tal como la de la Escuela de Trabajo Social de El Salvador, la de la Escuela de la Universidad Laica Vicente Roca fuerte de Guayaquil, así como de las escuelas chilenas, pero a su vez aclara lo siguiente:

Sobre la génesis y desarrollo del Taller en Costa Rica en las unidades académicas de Trabajo Social, se han tenido algunas ideas distorsionadas. Por lo general, se enfoca como un modelo copiado de experiencias de otras escuelas de otras latitudes. Si bien en su carácter más general considera los elementos básicos de todo taller en Trabajo Social, en su dimensión particular se diferencia de otras experiencias (p.44)

Dichasparticularidades conllevaron a que en Costa Rica, luego del III Seminario Latinoamericano de Escuelas de Servicio Social (Quito, Ecuador, 1971), se estableciera la necesidad de poner en cuestionamiento el currículum vigente, en el que se propone partir de una concepción de hombre y mujer particular, acercar el contenido formativo a las características del país y elevar el nivel científico. En síntesis se buscó adherir la educación al proceso de Reconceptualización del Trabajo Social (Molina y Romero, 1992).

A pesar de esos esfuerzos académicos, la formación "reconceptualizada" no logró (inclusive por las propias contradicciones de la misma) romper ese lastre conservador que le dio vida. Existieron intentos y debates, pero décadas después, se regresa a los causes del neopositivismo y las posturas modernizadoras del neofuncionalismo (Parra, 2005 y Bentura, 2006), así como algunas de sus expresiones tales como la teoría de sistemas.

Los elementos antes destacados son parte de la plataforma histórica que posibilitó la llegada de los talleres como la propuesta educativa más cercana a las aspiraciones de la época.Sin embargo, como se abordará más adelante existieron resistencias, críticas y autocríticas sobre este planteamiento pedagógico.

\section{Precedentes formativos del Taller en Costa Rica}

El tema de la formación de Trabajo Social en Costa Rica ha sido una discusión de casi setenta años, desafiando siempre la investigación producida hasta el momento sobre el tema. Desde el surgimiento académico que la caracteriza, propio de una compleja naturaleza intervencionista en la "cuestión social", asociado a la necesidad de establecer ligámenes entre las demandas profesionales y el asidero teórico que la relaciona con la legitimidad brindada por la política social, la 
profesión se ha ido forjando por una dinámica presión histórica que la entrecruza (proveniente de las fuerzas y movimientos sociales de clases),mediatizada desde luego por su alta organicidad con el Estado.

Comenzando en su fundación en 1942, las autoridades de la Escuela enfrentaron la necesidad de interrogarse sobre los soportes de la formación, prueba de ello, es lo destacado por Mora (1968) quien anota sobre este particular lo siguiente:

En 1944 se realizó, por parte de la Institución, un primer intento de discusión de los planes de estudio, dando participación a las entidades de bienestar social interesadas en la capacitación de su personal. Participaron en la discusión la Caja Costarricense de Seguro Social, el Patronato Nacional de la Infancia, la Secretaría de Trabajo y el Consejo Nacional de Prisiones. (p.20)

Para 1965, la Escuela en mención da un cambio en su estructura curricular en la que toma relevancia la llamada sistematización de la "metodología de la práctica". A su vez se incluyen cursos en que los (as) sujetos con que se trabajan son individuos, grupos y comunidades; y se establecen intervenciones formativas en instituciones, manteniéndose siempre el desvelo de la búsqueda de la integración de la relación teoría-práctica. Era evidente una preocupación importante por estrechar mayores relaciones con las instituciones sociales de la época, hallando ahí un argumento de legitimación, proyección y cumplimiento de los propósitos (a veces románticos) de la profesión en el marco del contexto costarricense y latinoamericano convulso, desigual y complejo.

El escenario de los años sesentas y setentas provocó una fuerte articulación en las Escuelas latinoamericanas de Trabajo Social, estimulando la convocatoria y celebración de actividades académicas que trataba entre otros, temas del escenario histórico, de las coyunturas regionales y desde luego de los contenidos formativos y los desafíos en la educación superior pública (Lima, 1981).

Tal y como se ejemplificó, para el caso de Costa Rica las reflexiones en materia formativa en la Escuela son erigidas desde la propia época de los cuarenta del siglo pasado, cruzando por replanteamientos interesantes en las décadas subsecuentes, con impulsos cada vez más relacionados con las contradicciones de los fundamentos que respaldaban al Trabajo Social costarricense, las exigencias del contexto, las condiciones institucionales desde donde intervenía, así como las expresiones de expoliación social que se manifiestan a lo largo del tiempo en la sociedad capitalista(Ver Fallas, 2010). Para 1971 la Escuela en estudio había celebrado su primer Jornada de Trabajo, donde definió bases epistemológicas, teóricas y metodológicas para sustentar su propuesta pedagógica, desembocando en una estructura curricular bajo la nomenclatura de "bloques". Guzmán (1978) explica que "la idea de los 'bloques' viene a ser una concepción primaria del taller, manteniendo los elementos de la trilogía intervención teoría-práctica, proceso pedagógico, servicio a los grupos populares y trabajo interdisciplinario; la práctica como eje central del proceso y otros" (p.45).

Vinculado a lo anterior, Alfaro,Arroyo, Araya, Jackson, Rosales y Villalta (1981), citan que las prefiguraciones del Taller se identifican en el Plan de Estudios de 1973, en el que la Escuela de Trabajo Social planteó los citados bloques. Estas autoras agregan también:

A partir de ese momento, se fueron delineando las bases teórico-metodológicas del modelo pedagógico del taller; a pesar de que en su etapa inicial era una estructura 
curricular basada en bloques: Metodología, Práctica, Realidad Nacional. La idea de 'bloques' viene a ser una concepción primaria del Taller, que supone la operacion alización de la trilogía Integración Teoría-Práctica, Integración Proceso Pedagógico, servicio a los grupos populares y trabajo interdisciplinario. La Práctica es el eje central del proceso. No obstante, se continúa trabajando bajo el sistema de cursos, pero a niveles integrados, porque se intenta impartirlos de acuerdo en cada nivel, en forma consultada, a través de reuniones de equipo (Alfaro y otras, 1981, pp.61-62).

Además, describen que esta propuesta tuvo sus limitaciones estructurales, organizativas y curriculares para ser desarrollada en la Escuela de Trabajo Social en la Ciudad Universitaria Rodrigo Facio. Se vislumbraron mejores condiciones en el marco de la inauguración de la Carrera de Trabajo Social en 1973, en lo que en aquellas décadas se conocía como Centro Universitario Regional de Occidente (C.U.O), y que responde de acuerdo a Türnnermann (1980), al inicio de un proceso de expansión cualitativa y cuantitativa de la educación superior en el país y América Latina en general. Alfaro y otras (1981, señalan al respecto:

El surgimiento de los Centros Universitarios obedece a una política de descentralización de la Universidad de Costa Rica. Fue a partir del II Congreso Universitario, efectuado en agosto de 1966, que cobró fuerza la idea de su creación, pues a mediados de la década de los sesenta la Universidad empieza a buscar solución a varios problemas, cuyos planteamientos sugerían la necesidad de diversificar la enseñanza superior [...] En 1968 se aprobó por unanimidad, la creación de los Centros Regionales de San Ramón y Liberia, según acta de la sesión n. 1619 del Consejo Universitario celebrado el 12 de enero de ese mismo año. (p.37)

Los cambios que experimenta la Universidad de Costa Rica en la década sesenta y setenta del siglo XX en política de descentralización constituye un factor positivo para la Escuela de Trabajo Social, al propiciarse también el espacio para impulsar la propuesta de los Talleres (Alfaro y otras, 1981). Estas autoras agregan:

Es importante recalcar que junto con lo anterior se dieron las condiciones históricas necesarias, ya que la política de descentralización de la Educación Superior y el proceso de Reconceptualización del Trabajo Social proponen a la Escuela de Trabajo Social de la Sede la necesidad de replantear tanto los propósitos, objetivos y objeto de la profesión, como las funciones que debían cumplir los profesionales que formaba. Con ello, se vio en el C.U.O la posibilidad de desarrollar programas nuevos y flexibles, a través de los cuales se podía lograr la formación de un profesional más consciente y comprometido con los sectores campesinos pues hasta el momento la Escuela se dirigía hacia las zonas urbanas y semiurbanas del Área Metropolitana. Además se vencieron una serie de obstáculos para abrir la Carrera originados por la carencia de recursos financieros, humanos y materiales. A nivel de Centro Regional y de quienes apoyaron la apertura de la carrera se tuvo que mantener una actitud crítica frente al respaldo ofrecido por algunas instituciones de Bienestar Social, que dieron a conocer a la Universidad de Costa Rica el interés por la Carrera. (p.102)

Las condiciones histórico-sociales de los años sesentas y setentas fueron una mediación deteminante en la configuración del Taller en el país, aunado a las mutaciones que experimentó la uni- 
versidad pública latinoamericana y las agendas no resueltas en la formación en Trabajo Social, canalizadas en la idea de la articulación más estrecha en la teoría con la práctica, en el alejamiento y rechazo al Estado como síntesis de los intereses capitalistas y de dominación de clase, de liberar al pueblo latinoamericano del yugo explotador de sus oligarquías, como del imperialismo norteamericano, junto con un mayor acercamiento a prácticas "científico-epistemológicas" que se sublevaban contra las formas tradicionales de pedagogía que eran problematizadas por los postulados de la llamada Educación Popular.

\section{El giro pedagógico de 1976}

Para 1974, se celebró el IV Seminario Latinoamericano de Escuelas de Servicio Social "Organización y sistematización de las prácticas profesionales" en San José, Costa Rica, con representantes de Argentina, Brasil, Chile, Colombia, Ecuador, Panamá, Guatemala, Honduras, El Salvador, México, República Dominicana, Perú, Venezuela y Costa Rica. En el marco de este seminario se impulsa con mayor determinación el planteamiento del Taller como propuesta pedagógica para las instancias educativas relacionadas con esta profesión en América Latina.

En ese evento la delegación costarricense expone a su vez una serie de aportes que posteriormente se integrarán al giro pedagógico, organizativo, evaluativo y didáctico que significó esta propuesta. Por ejemplo entre las ponencias nacionales que se registran en ese acontecimiento se discutieron asuntos tales como la reformulación del "plan de acción" de la Escuela, la necesidad de sistematizar las experiencias prácticas así como la innovación pedagógica (Colegio de Trabajadores Sociales de Costa Rica,1974). Al mismo tiempo, se insistió en la necesidad de profundizar en lo referente al Taller como "método" pedagógico y propiciador de la triple integración: teoríapráctica, investigación-docencia-acción social, así como de concatenar las relaciones estudiantesdocentes y los llamados grupos populares.

En ese seminario también se propusieron intervenciones integradas por diversos niveles de carrera, especialmente en zonas rurales, bajo la supervisión de equipos de docentes de distintas áreas, tales como Sociología, Filosofía, Psicología, Historia, Economía y Trabajo Social (Campos y otras, 1977). El planteamiento que caracterizó la formación de Trabajo Social en esos años señalaba que las experiencias de Taller, en sus primeros niveles de carrera, buscaban que el o la estudiante tuviera una visión general de su campo de especialización y de la propuesta pedagógica en mención. A su vez, se intentaba evidenciar la diferencia de elementos teórico-metodológicos según el nivel de carrera, como también alcanzar un proceso de inserción en un cierto lugar en el tiempo y el espacio, junto a un grupo de compañeras (os), buscándose finalmente una acción social que se guiara por el análisis de la realidad, acompañada de un proceso de sistematización de la experiencia.

Parte del llamado fundamento "teórico-metodológico" que acompañó a las experiencias de talleres, se centraba especialmente en la llamada investigación-acción, la cual para Campos y otras (1977), es una concepción metodológica que articulaba el proceso de formación profesional al de producción de conocimientos. Posteriormente, entre 1974 y 1975 se desarrolló la VI Jornada de Reflexión y Evaluación de la Escuela de Trabajo Social, donde se demandaba la necesidad de continuar con la búsqueda de un currículum cuya base fuera la integración y la perspectiva crítica. Se brindaron recomendaciones para reestructurar el plan de estudios sobre la base de la integración antes descrita, recurriendo en 1976 al Taller como sustento pedagógico. A su vez, se determinaron 
las competencias de la estructura organizativa de la Escuela, y se plantearon las recomendaciones vinculadas a la interdisciplinariedad en la formación. Una síntesis de ese plan es expuesto a continuación:

\section{Cuadro 1}

\section{Plan de Estudios(1976-1981 2)}

Objeto de Estudio: Se estudian los grupos sociales que presentan un marcado deterioro en Costa Rica, principalmente los populares, que por ser históricamente los grupos de atención mayoritaria, cualitativa y cuantitativamente de la profesión, obligan a estudiantes y profesionales en Trabajo Social a manifestar compromiso o identificación con ellos.

Objetivos de la Escuela: Formar un profesional con capacidad para comprender científicamente la realidad y para colaborar en los procesos de transformación social estableciendo relaciones dialógicas con los grupos populares, y que ejecute acciones de asistencia y promoción social conjuntamente con otros profesionales, atienda situaciones de asistencia y promoción social conjuntamente con otros profesionales; asesore, planee, ejecute y evalúe acciones, proyectos y programas de Bienestar y Desarrollo Social; diseñe y realice investigaciones sociales y supervise personal asignado a programas de Bienestar Social.

Organización Curricular: método de Taller, el cual es un proceso de enseñanza-aprendizaje de carácter social: en el que se transmite la información y síntesis del desarrollo científico de la humanidad. Se organiza curricularmente en forma de unidades integradas de conocimiento, extraídas de la realidad social y abordadas desde la perspectiva científica, que partiendo de la experiencia empírica (práctica académica) reconoce un camino que va de lo simple a lo múltiple para retomar la realidad y contribuir a su transformación. La organización curricular presupone la integración de tres niveles: integración teoría-práctica, integración docenciainvestigación y acción social, integración docente-estudiante-grupos sociales. Contenidos teóricos y prácticos en tres áreas temáticas: área de Teoría Social, área de Realidad Nacional, área de Teoría y Metodología del Trabajo Social.

Este plan estaba inspirado en un proceso de formación cuyo fuerte era el trabajo de campo estimulado por problemas generadores, el logro de objetivos por programas y proyectos, la participación activa del estudiantado en todas las fases del proceso de formación (incluyendo la programación y evaluación), el desarrollo de una organización tendiente a la producción y difusión del conocimiento, el alcance de objetivos orientados al desarrollo de saberes, habilidades y actitudes para el cambio social, así como una docencia interdisciplinaria. En lo que respecta a los problemas generadores cuyo papel era trascendental en la direccionalidad de los Talleres, Molina y Romero (1992) detallan lo siguiente:

Los problemas generadores responden a una escala de complejidad que va, de una caracterización general de los grupos sociales populares y su contexto, pasando por las diferentes formas organizativas que los agrupa en la satisfacción de sus necesidades sociales, hasta llegar a las instituciones de bienestar social, cuyas políticas tienen que ver con la satisfacción de las necesidades de los grupos sociales en cuestión. El proceso

2 El mismo recibe modificaciones de tipo administrativo que se aprueban en el año de 1981. Fuente: Molina y Romero, 1992 , p.71 
teórico-práctico consiste en apoyo teórico y trabajos grupales, alrededor de temas relacionados con el problema generador intra-aulas y el de trabajo de campo, con inserción en comunidades, organizaciones o instituciones, según sea el nivel de Taller, ambos momentos deben estar íntimamente relacionados, retroalimentándose uno al otro. (pp.70 y 71)

El currículo estaba organizado en cinco años, el primero correspondía a los Estudios Generales y otras materias consideradas como requisitos para ingresar a la carrera. Entre estas materias estaban: la actividad cultural (en la que se recomendaba taller de teatro de títeres, taller de teatro, danza folclórica o apreciación del folclor), los cursos integrados de humanidades, repertorio de ciencias, matemática básica, elementos de economía, seminarios de realidad nacional I y II, así como un semestre de introducción al Trabajo Social. En segundo año únicamente se matriculaban el Taller I, en tercero el Taller II, en cuarto el Taller III y en quinto el Taller IV (Escuela de Trabajo Social, 1979).La lectura del contexto y su articulación con la propuesta de formación profesional que subyacía en este nuevo plan de estudios se resume en el Cuadro 2 (Ver Apéndice).

La organización de los talleres se gestaba por cuatro fases que adquirían sentido a lo largo de los años, las mismas son explicadas a continuación. En cada taller que se impartía se particularizaban estas fases tratando de responder al nivel de carrera, el espacio de aprendizaje según complejidad y el conocimiento adquirido.

\section{Cuadro 3}

\section{Fases de los Talleres del Plan de Estudios (1976-1981)}

\section{Descripción general}

Fase introductoria: Esta fase pretende que el o la estudiante adquiera una visión general del Trabajo Social como disciplina y comprensión del modelo pedagógico y de los conocimientos que profundizará a lo largo de su carrera.

Fase instrumentalización: Se pretende que el estudiantado asimile y corrobore elementos teóricos y metodológicos que le faciliten sucesivas aproximaciones al abordaje de la realidad en estudio. Se encamina hacia una definición del objeto de estudio, categorías a investigar, selección de técnicas de investigación, planificación, intervención, instrumentos de registro y manejo de fuentes secundarias.

Fase de trabajo de campo: Se trata de la inserción del estudiantado en diversas zonas geográficas seleccionadas bajo criterios tales como: concentración en la zona de grupos populares, oferta de trabajo sin desplazar mano de obra, posibilidades de alojamiento y alimentación. Los estudiantes se organizan en grupos durante el período de práctica, con el propósito de hacerle frente a las tareas de inserción investigación-acción y a la sistematización. Se pretendía fomentar en los estudiantes un proceso de organización cooperativa, así como vivenciar las condiciones de vida de los grupos populares (entendidos como aquellos que se encuentran ubicados en los niveles más bajos de la escala social. Son trabajadores manuales que en unos casos no son dueños de los medios de producción y que en otros lo son pero el producto de su trabajo a penas les permite sobrevivir). Esta aproximación vivencial permitía sentir y percibir lo exterior, alcanzando progresivamente niveles de profundización.El grupo estudiantil y el docente tenían la responsabilidad de captar la realidad, 


\begin{abstract}
orientándose con la teoría existente para que ambos logren interpretar los fenómenos en estudio y de esta manera alcancen mayores niveles de conocimiento. Tal situación se posibilitaba a través de las sesiones de supervisión semanal en el campo y a través de los Seminarios de Implementación Teórica, ya que es, en esta instancia, en donde el análisis y la reflexión de situaciones concretas señalan el rasgo dominante de esta fase. Las supervisiones en el campo se llevan a cabo con el apoyo de 2 o 3 profesores. Estas se caracterizan por una participación conjunta docente-estudiante, en la programación y ejecución del trabajo. En las supervisiones participan varios profesores de distintas materias, tienden a concentrarse en uno o dos días y son una respuesta a la necesidad de superar deficiencias teóricas y/o metodológicas detectadas en la ejecución del trabajo de campo.Debido a que tal experiencia presupone que deberá ser sistematizada, requería entonces ser planificada con objetivos claramente definidos, ya que es la acumulación de conocimientos emergentes de la experiencia de campo, lo que da origen a la sistematización. Por tales razones los grupos de estudiantes en programan en conjunto e individualmente su quehacer.
\end{abstract}

Fase sistematización: Se perseguía con ella que el estudiante logre ordenar, organizar e interpretar su experiencia en el trabajo de campo, experiencia que se cimentaba en elementos teóricos y metodológicos elementales para investigar las fuentes que le permitieron abordar una realidad concreta, en un tiempo determinado. Al ordenar, organizar e interpretar la información obtenida, los grupos de estudiantes deben realizar una exposición escrita de sus investigaciones y acciones (especie de tesinas o informes); tal documen-to reproducirá el conocimiento adquirido de la realidad, así como los aspectos metodológicos empleados y elaborados a través de esa práctica, y finalmente los aspectos organizativos del equipo de estudiantes.

Fuente: Elaboración propia a partir de Escuela de Trabajo Social (1979), pp.3-5

Como se aprecia, la crítica y la autocrítica de la formación profesional han sido características del proyecto formativo original de la Escuela. Sin embargo, su naturaleza y objetivos no necesariamente se vinculaban más allá de una orientación reformista romántica o desarrollista de la época, que a su forma, intentaba dar respuesta al contexto nacional, así como plantear la interlocución tanto con distintas áreas del conocimiento como con los llamados sectores populares. El principal eje crítico que caracterizó este debate fue el tema de la relación teoría-práctica, así como el operacionalizar un discurso ideológico y político que se vinculaba con influencias de la educación popular, la Teología de la Liberación, la Investigación Acción Participativa, el Maoísmo, el Existencialismo y el Estructuralismo, así como los planteamiento de lo que en Trabajo Social se denominó epistemológicamente Método Básico (ver Lima y Rodríguez 1977; Araya y otros, 1988, así como Molina y Romero, 1994).

Aunado a ello aparecía la inconformidad a la fuerte dependencia laboral de esta categoría profesional con el Estado capitalista, tanto como la propuesta de que el significado real de la profesión adquiría sentido, primordialmente, al vincularse con las llamadas organizaciones populares, campesinas, obreras, indígenas, de mujeres, ambientalistas o no dependientes de este ente, las cuales supuestamente, no representaban intereses antagónicos, la hegemonía burguesa occidental, los intereses de las oligarquías políticas, ni la agenda del capitalismo local, siendo esta la interface de la relación teoría-práctica (ver Montaño, 1998). Sin duda, estos esfuerzos y argumentos son parte de uno de los momentos más intensos de reflexión educativo-ideológico-políticos que se identifican en la historia del Trabajo Social costarricense del siglo pasado. 
Tal voluntad universitaria no se libró de las limitaciones propias de las universidades tradicionales, de las formas de enseñanza, aprendizaje más hegemónicas, así como de la poca sincronía con profesionales docentes de otras áreas, aunado a los altos costos operativos de esta modalidad, y desde luego, los riesgos de diferente naturaleza que sufrían docentes y estudiantes internándose en zonas rurales o lejanas de sus lugares originales de convivencia, situación que también les reducía sus lazos familiares, afectivos o cumplimiento de otras responsabilidades como cuido de hijos e hijas, familiares con enfermedades, personas adultas mayores, y desde luego trabajar para asistir sus estudios con algún ingreso. De acuerdo a Mora (1968) y Mora, Murillo, Oviedo y Segura (1978), este estudiantado provenía mayoritariamente de sectores medios, bajos, con familias asociadas a empleos en actividades económicas en las ramas del comercio, la artesanía, la agricultura, la industria y los servicios, con una trayectoria genealógica poco o nada relacionada con la educación superior.Dentro de las limitaciones que se presentaron para el desarrollo del Taller se identifican las siguientes:

\section{Cuadro 4}

\section{Limitaciones para la implementación del Taller en el Trabajo Social en Costa Rica}

1. Falta de reflexión y análisis de los lineamientos y postulados esenciales del Taller (crítica y autocrítica de sus insuficiencias)

2. Marcado eclecticismo producto de las numerosas ideologías que coexistían en forma yuxtapuesta.

3. Tendencia a un metodologismo.

4. No resolver las separaciones entre teoría y práctica tal y como se argumentaba en sus postulados.

5. Equipararlo con la estructura formal y evaluativa de la UCR.

6. Las demandas de formación a los cursos de otras unidades, y que no sean bajo esta modalidad.

7. La formación en educación primaria y secundaria de los docentes y estudiantes que es básicamente bancaria, así como los otros cursos que se recibían en la Universidad.

8. La resistencia de participación de cada una de las disciplinas que se identifican en el taller (Psicología, Filosofía, Estadística, Sociología, entre otras), así como la escasa experiencia en esta pedagogía por parte de otras unidades académicas.

9. Imposibilidad de trabajar por parte de las personas que estudian, ya que la experiencia requiere de tiempo completo.

10. La forma de evaluación demanda algunas acciones conductuales y memorísticas, que se contraponen a la esencia del taller, junto a una presencia de sobrevaloración de la práctica.

11. Enfrentamiento de esta propuesta con las personas que venían con un plan de estudios diferente de generaciones anteriores.

12. La integración de la docencia, la investigación y la acción social en el Taller, no logra concretarse en estos años plenamente, ya que las actividades académicas de cada Sección de la Escuela de Trabajo Social no responden en su totalidad a un proyecto integrador.

Fuente: Elaboración propia a partir de Campos y otras, 1977 y Mora y otras, 1983. 
A lo largo de este último apartado, se logró identificar, cómo la influencia histórica del continente latinoamericano, generó presiones sobre la formación profesional. El llamado Movimiento de Reconceptualización, fue prueba de esa complejidad. Las luchas y resistencias hacia los rumbos que se tomaban para responder a esas condiciones fueron particulares y singulares en cada país, en el caso costarricense, pareció ser una base para replanteamientos curriculares, didácticos y pedagógicos.

La formación también entró en este período en un espacio de madurez académica, donde su interlocución con otras áreas por medio de la creación de la Facultad de Ciencias Sociales es un hecho de suma relevancia. Otro de los insumos que caracterizan esos años y que aún debe investigarse, es la llamada influencia de la dialéctica como sustento teórico-metodológico, que amplía el variopinto que sincréticamente se manifestaba en esta profesión.

El aparecimiento del Taller, como estrategia pedagógica, marca el rumbo de lo que antes eran los espacios de práctica supervisada y que hasta la primera década del siglo XXI es un eje base del cuerpo colegiado de esta unidad. Los años en estudio también dan evidencia de la necesidad de espacios sostenidos de autocrítica y crítica en la formación, insumo que permitió, en alguna medida, ventilar cuestiones que se requieren debatir de manera sostenida.

\section{A guisa de cierre}

La madurez de la formación profesional en Trabajo Social, se ha ido forjando entre procesos con intentos de ruptura. Algunos que concluyen en modernizar o reformar el corpus académicoprofesional con una fuerte intensión de dar respuesta a las demandas del contexto nacional y latinoamericano. La incorporación de la Escuela de Trabajo Social a la UCR, permitió un impulso reflexivo sobre su significado en el mundo académico y profesional, así como los desafíos que ello implica, creando y recreando históricamente algunas propuestas para atender esas necesidades.

La formación en este campo ha demostrado ser objeto de análisis y discusión en el país desde la década de 1940, llevando a constantes cambios y replanteamientos. En todo el trayecto histórico analizado, el desvelo por integrar la teoría y la práctica, así como el llamado a sistematizar para "producir teoría", fueron características en el desenvolvimiento de la formación académica y sus propuestas más determinantes como el Taller. Esta innovación pedagógica fue sin duda la propuesta más determinante desde los inicios de la formación y que, con sus contradicciones, conjugó preocupaciones que se fueron gestando a lo interno de la Escuela, asociadas con ciertos acercamientos y distancias al debate latinoamericano del Trabajo Social, las Ciencias Sociales y las propuestas de los años setentas en otros campos como la Educación, la Teología, la Psicología y la Sociología, para citar algunas.

Un punto medular en esta propuesta fue la necesidad de evitar la verticalidad en el aprendizaje, no sólo entre docentes y estudiantado, sino también con las personas con las que se relacionaba este tipo de prácticas y se ubicaban bajo la nomenclatura de "grupos populares". Se reconoce a su vez que esta iniciativa se acercaba a desafiar la integración peculiar de la estructura más típica de las universidades públicas latinoamericanas, compuestas por separados procesos de investigación, docencia y extensión. 
A partir de lo expuesto es evidente que el Movimiento de Reconceptualización fue un espaldarazo de este ideal, al parecer de origen chileno, y configurada a su modo en cada país. La propuesta del taller tuvo eco en Costa Rica en una coyuntura de la unidad académica que se estaba examinando tanto en su contenido académico como organizativo. Otro logro parcial fue el aglutinar un equipo interdisciplinario que osadamente acompañara este trabajo. La importancia de estimular la sistematización también se realizó en tanto permitía reflexionar y evidenciar las intervenciones realizadas, sin olvidar la crítica al presupuesto de que teorizar y sistematizar son esfuerzos intelectuales de marca diferenciada.

El acercamiento y convivencia del estudiantado a la vida cotidiana de ciertas fracciones sociales, fue un vigoroso trabajo, sin embargo la desmediatización que de ahí surgía, imposibilitaba recuperar aquellos complejos que entraman al Trabajo Social con las sociedades capitalistas de las que forma parte, a saber: las oscilaciones del modo de producción y sus crisis permanentes, las manifestaciones de la "cuestión social" que se priorizan por parte de las clases hegemónicas para ser intervenidas instrumentalizando las políticas públicas de cuño social, que son en definitiva el objeto definitivo de la legitimación de la profesión en América Latina, las cuales se viabilizan en las instituciones que contratan asalariadamente a profesionales de esta rama, delimitando sus atribuciones, estableciéndole límites, impulsándole ciertas competencias y demarcándole un radio de trabajo que transmuta a lo largo de la historia, a partir del movimiento de las fuerzas sociales que avanzan y retroceden en la seguridad, la asistencia, la protección y la compensación social.

Para nuestros días la Escuela de Trabajo Social ha vuelto a retomar el debate de las prácticas en la formación, justamente el poder hacer este valioso recorrido por la propuesta de los talleres propicia que las discusiones tengan un asidero más articulado y diferenciado con los propios esfuerzos históricos que lideraron cuadros docentes del siglo pasado. 


\section{Referencias}

Alfaro, Lidia; Arroyo, Carmen; Araya, Rosa; Jackson, Guiselle; Rosales, Rosa; Villalta, Olga. (1981). Bases teórico-metodológicas del taller en Costa Rica y resultados de su aplicación: un estudio comparado. Seminario de Graduación para Licenciatura en Trabajo Social, Universidad de Costa Rica. San José, Costa Rica.

Araneda, Luis. (2005). La Reconceptualización del Trabajo Social latinoamericano. Su análisis y prospectiva. En Norberto Alayón (Org.), Trabajo Social latinoamericano: a cuarenta añosdelaReconceptualización(pp.149-163).BuenosAires,Argentina:EspacioEditorial.

Araya, Rosa; Calderón, Manuel; Chinchilla, José y Vega, Cecilia. (1986). Análisis del surgimiento y desarrollo del Servicio Social en Costa Rica y su ubicación en el contexto económico socialypolítico.Informedeinvestigación.Alajuela,CostaRica:UniversidaddeCostaRica.

Aylwin, Nidia y Gissi, Jorge. (1976, $2^{\circ}$ cuatrimestre). El taller: integración de teoría y práctica. Selecciones del Servicio Social, 20(4), 19-38.

Barboza, María. (1977). Une expérience de la méthode active d'enseignement a l'école de Travail Social de l' Université de Costa Rica. (Tesis de Maestría nopublicada), Université de Paris VIII - VINCENNES, Francia.

Beeche, Héctor. (1951). Servicio Social Criminológico. La Habana, Cuba: Ed. Jesús Montero.

Bentura, Pablo. (2006). Ofensiva neoconservadora: impacto en las auto-representaciones del Servicio Social. En Margarita Rozas (Org.), La formación y la intervención profesional: Hacia la construcción de proyectos ético-políticos en Trabajo Social (pp.85-96). Buenos Aires, Argentina: Espacio Editorial.

Campos, Ivette; Molina, Emilia; Molina, María; Romero, Carmen y Ruiz, Ana. (1977). Evolución social de la estructura académica de la Escuela de Trabajo Social en Costa Rica. (Tesis de Licenciatura en Trabajo Social no publicada), Universidad de Costa Rica.

Casas, Gerardo. (1975). Evolución histórica del Servicio Social costarricense. (Tesis de Licenciatura en Trabajo Social no publicada), Universidad de Costa Rica.

Centro Latinoamericano de Trabajo Social. (1988). Sistematizando experiencias de Taller en las escuelas de Trabajo Social de América Latina. Lima, Perú: Autor.

Colegio de Trabajadores Sociales de Costa Rica.(1974). Boletín de Información para trabajadores sociales, 3 (14).

De León, Tomasa. (2005). Movimiento de Reconceptualización: aportaciones sobre el tema. En Norberto Alayón (Org.). Trabajo Social latinoamericano: a cuarenta años de la Reconceptualización (pp.195-210). Buenos Aires, Argentina: Espacio Editorial. 
Escuela de Trabajo Social.(1979). Talleres: Plan de estudios, características y estructuras. San José, Costa Rica: Universidad de Costa Rica.

Esquivel, Freddy. (2002). Génesis reproducción del Trabajo Social en Costa Rica. (Tesis de Maestría en Trabajo Social no publicada), Universidad de Costa Rica.

Esquivel, Freddy. (2007). Trabajo Social en Costa Rica: del ideario liberal a su constitución en el reformismo. San José, Costa Rica: Editorial UCR.

Fallas, Yessenia. (2010). Fundamentos teórico-metodológicos en el Trabajo Social costarricense 1942-1970: un análisis a partir de sus mediaciones constitutivas. (Tesis de Maestría en Trabajo Social no publicada). Universidad de Costa Rica.

Guzmán, Laura. (1973). Una estrategia para el cambio en el Trabajo Social Costarricense y de la sociedad: aspectos tácticos, metodológicos y estructurales. (Tesis de Licenciatura en Trabajo Social no publicada). Universidad de Costa Rica.

Guzmán, Laura. (1978). El taller: una alternativa pedagógica innovadora para la formación de trabajadores sociales. San José, Costa Rica: Editorial Trabajo Social.

Lima, Leila. (1980). Desarrollo del Trabajo Social en América Latina. Revista Acción Crítica8, 25-39.

Lima, Leila y Rodríguez, Roberto. (1977, Julio). Metodologísmo: estallido de una época. Revista Acción Crítica, 2,15-41.

Molina, Lorena y Romero, María. (1992). La planificación y la evaluación curricular: la experiencia de la Escuela de Trabajo Social de la Universidad de Costa Rica 1942-1991. En Alicia Guardián, Laura Guzmán, Lorena Molina y María Romero. Planificación y Evaluación Curricular en Trabajo Social (pp. 75-173).San José, Costa Rica: ALAETS- CELATS-Alma Mater.

Molina, Lorena y Romero, María. (1994). Costa Rica, la educación en Trabajo Social. Cuadernos de Trabajo Social, 7,11-41.

Molina, Lorena y Sáenz, Jorge. (1994, Octubre). Trabajo Social: lo académico y lo profesional. Revista de Ciencias Sociales, 64,101-107.

Mora, Gerardo; Castro, Silvia; Araya, Rosa y Vega, Cecilia. (1983). La carrera de Trabajo Social en el Centro Regional de Occidente: una visión crítica del taller. Serie Documentos. Alajuela, Costa Rica: Universidad de Costa Rica.

Mora,Rosa.(1968). Algunos aspectos relacionados con la formación de profesionales en la Escuela de Servicio Social de la Universidad de Costa Rica. (Tesis de Licenciatura en Ciencias Económicas no publicada), Universidad de Costa Rica. 
Mora, Virgilio, Murillo, Dinorah, Oviedo, Rosa, Segura, Zaida. (1978). Análisis de los factores socioculturales que contribuyen en la elección de la carrera de Trabajo Social en la sede central y centros regionales. (Tesis de Licenciatura en Trabajo Social no publicada), Universidad de Costa Rica.

Parra, Gustavo. (2002). Los proyectos socio-profesionales en el Trabajo Social argentino: un recorrido histórico. En José Paulo Netto, Gustavo Parra, Alfredo Carballeda, José Coraggio, Nora Aquín, Mario Robirosa, María Felicitas, Carlos Eroles, y Adriana Clemente. Nuevos escenarios y práctica profesional: Una mirada crítica desde el Trabajo Social (pp.31-51). Buenos Aires, Argentina: Espacio Editorial.

Quezada, Margarita. (1995). El Contexto Social de los 70. En Margarita Quezada, Teresa Matus, Nelda Rodríguez, Leonardo Onetto, Malvina Ponce de León y Dolly Paiva. Perspectivas Metodológicas en Trabajo Social. Lima, Perú: CELATS.

Retana, Juan. (2002). As dimensões a ética e da política no Serviço Social: A particularidade a Costa Rica. (Tesis de Maestría en Trabajo Social no publicada), Universidad Federal de Río de Janeiro, Brasil.

Romero, María. (1988). El Taller de la Escuela de Trabajo Social de la Universidad de Costa Rica: antecedentes, desarrollo y resultados. 1976-1986.(Tesis de Maestría en Educación no publicada), Universidad de Costa Rica.

Türnnermann, Carlos. (1980). Pensamiento universitario centroamericano. San José, Costa Rica: EDUCA.

Yazbek, María. (2000). Políticas sociales y asistenciales: estrategias contradictorias de gestión estatal de la pobreza de las clases subalternas. En Elisabete Borgianni y Carlos Montaño (2000). La política social hoy, pp. 110-135. São Paulo, Brasil: Editora Cortez. 


\section{Apéndice}

\section{Cuadro 2}

\section{Fundamentos del Plan de Estudios (1976-1981)}

Contexto nacional: Costa Rica es una nación subdesarrollada, en una situación de dependencia externa que afecta a los grupos populares. En nuestro país esta dominación se ejerce principalmente a través de la educación, las políticas de bienestar social, los medios de comunicación. Tal condición se manifiesta cuando se analizan las estructuras agrarias e industriales, de poder y de las clases sociales. Frente a esa condición se asume la necesidad de la liberación del hombre. Por tanto se requiere que la formación propicie el estudio de la sociedad costarricense, dentro del contexto latinoamericano y mundial, del Trabajo Social en su relación actual a partir de elementos de la Ciencia Social que ayudan a su interpretación.

Perfil de Trabajo Social: Agente que, siendo externo, se inserta en la situación concreta para colaborar en la creación de condiciones más racionales que favorezcan la toma de conciencia, por parte del hombre dominado, su capacitación y organización.

Fin y propósitos de la formación: Formación es definida por la realidad en que está inserto el estudiante y por los fines propios de la Universidad. Se busca el desarrollo de la capacidad para conocer y comprender la sociedad de forma científica (en su dinamismo y unidad), así como fortalecer habilidades para crear y utilizar métodos, técnicas e instrumentos que permitan colaborar en los procesos de transformación social. Sumado a gestar actitudes que lleven a comprometerse responsablemente en la realización de acciones concretas mediante el establecimiento de relaciones dialógicas con los sectores populares, a partir de necesidades de grupos, comunidades, organizaciones $y / 0$ instituciones, asociadas a los fines de la transformación social.

Fundamentos teórico-metodológicos: Un concepto de hombre según el cual éste es sujeto de su transformación y del mundo en que vive. Una concepción de realidad como unidad dinámica (dialéctica en el sentido de que constante cambio se genera, a partir de la oposición de los elementos que se dan en situaciones concretas y de acuerdo a ciertas leyes)Concepción del conocimiento como proceso que se da a partir del elemento sensorial y concreto para llevar a la elaboración abstracta y luego, a una interpretación teórica de la realidad concreta.

Habilidades estimuladas por la formación: La acción convenientemente fundamentada, reflexionada y sistematizada, permite lograr un conocimiento más dinámico y profundo sobre la realidad social. Para ello se requiere la observación, comprensión e interpretación científica de los fenómenos sociales a partir de situaciones concretas. Definir políticas generales, formular y ejecutar planes, de acción (delimitación de fines, objetivos, estrategias y tácticas, toma de decisiones y trabajos en equipo).Aplicación creativa y racional de métodos, técnicas e instrumentos propios del Trabajo Social (concienciación, capacitación, organización y gestión). Sistematización de la experiencia práctica.

Fuente: Elaboración propia a partir de Campos y otras, 1977. 
